\title{
A BSDE with Delayed Generator Approach to Pricing under Counterparty Risk and Collateralization
}

\author{
Francesco Cordoni ${ }^{1}$ and Luca Di Persio ${ }^{2}$ \\ ${ }^{1}$ Department of Mathematics, University of Trento, Via Sommarive 14, 38123 Trento, Italy \\ ${ }^{2}$ Department of Computer Science, University of Verona, Strada le Grazie 15, 37134 Verona, Italy \\ Correspondence should be addressed to Luca Di Persio; dipersioluca@gmail.com
}

Received 30 March 2016; Revised 18 May 2016; Accepted 1 June 2016

Academic Editor: Jiongmin Yong

Copyright (C) 2016 F. Cordoni and L. Di Persio. This is an open access article distributed under the Creative Commons Attribution License, which permits unrestricted use, distribution, and reproduction in any medium, provided the original work is properly cited.

We consider a nonlinear pricing problem that takes into account credit risk and funding issues. The aforementioned problem is formulated as a stochastic forward-backward system with delay, both in the forward and in the backward component, whose solution is characterized in terms of viscosity solution to a suitable type of path-dependent PDE.

\section{Introduction}

Starting from the spreading of the credit crunch in 2007, empirical lines of evidence have shown how some aspects of financial markets, neglected up to that point by theoretical models, are instead fundamental in concrete economical frameworks. In particular, let us mention the violation of standard nonarbitrage relation between forward rates and zero-coupon bonds. Even though we will not address the latter problem in the present paper, we would like to underline how it is very connected to the topic we will treat, as witnessed by the recent, wide, and growing literature linked to the socalled multicurve modelling; see, for example, [1-5] and the references therein.

In what follows, we will focus on a different issue which emerged after the last financial crisis, namely, the problem of pricing derivatives contracts, including the possibility of the counterparty default, that is, the event in which a borrower fails to make the required payments to his lender. Such an event is treated in the framework of the credit risk which, according to [6], is defined as the potential that a bank borrower or counterparty will fail to meet its obligations in accordance with agreed terms. Even if the number or type of related financial losses is rather huge, it is interesting to note that they may be complete, as in the case of default, or even partial and can happen in a number of different cases, for example, if a consumer fails to make a payment related to a line of credit or if an insolvent insurance company does not pay previously stipulated policy obligations or a bank that because of its insolvency does not return funds to a depositor. We would like to underline the notion that the credit default risk has a great impact on almost all the credit-sensitive transactions, also including mortgages, loans, securities, and derivatives. Hence, its careful determination and forecasting are crucial tasks, especially in the modern theory of financial markets (see, e.g., [7, 8]), which are widely characterized by sophisticated contracts of the aforementioned type. In particular, the wrong estimation of credit default risk that, at different levels, has been experienced at the end of the last decade is intrinsically linked to the inadequacy of classical models in describing real financial markets, mainly because of the unrealistic hypotheses of the existence of a unique risk-free rate, that is, the theoretical rate of return of an investment with zero risk, or the possibility of having unlimited access to funding. Our aim is to derive a mathematical formulation of such problems, while we refer the interested reader to, for example, $[9,10]$ and references therein, for a deep study of related financial implications; see also [11], where the credit risk is studied in connection with the so-called Catastrophe Bonds, [12] where the default probability problem for credit risk is considered, [13] which concerns a large deviation approach, and [14] where related trigger prices determination for convertible contingent bonds is treated. 
Recently, several works appeared that try to include counterparty risk, that is, the risk to each party of a contract that the counterparty will be unable to meet contractual obligations, as well as funding issues in pricing financial contracts, leading to a systematic treatment of both of them. In particular, to our knowledge, the first attempts in the direction of developing a concrete framework able to treat both the counterparty risk and the funding constraints can be found in [15-17]. However, we will mainly refer to a slightly different and yet closely related approach, namely, the one developed in $[9,18]$. Let us mention that both approaches identify backward stochastic differential equations (BSDEs) as a fundamental mathematical object to consider a financial setting characterized by counterparty risk; see, for example, $[19,20]$.

In what follows, we also exploit the approach developed in $[18,21]$, where the authors firstly consider the present value of a contract as the discounted present value of future payoffs, and then include margin variations and counterparty risk in their valuation BSDE, which turns out to be risk-free rate independent. In fact, the latter depends only on different funding rates which are directly observable on the markets.

The main contribution of the present work is thus to give rigorous and general mathematical foundation of the previously introduced setting. In particular, following [18, 21], we will consider the so-called master pricing equation generalizing it in several directions. Firstly, we will consider possibly path-dependent hedging strategy exploiting the so-called path-dependent calculus developed in [22,23]. Secondly, we will not assume any differentiability assumptions in order to consider viscosity solutions for the related pricing PDE. Finally, as major generalization, we consider a margining procedure that can be path-dependent with respect to the portfolio. In fact, as pointed out in [15, Remark. 5.5], in real world, the margining scheme often depends upon its past values. It is worth mentioning that the latter is highly nontrivial, since, from a mathematical point of view, it implies that the related BSDE generator depends on its past values as well.

Let us recall that the first rigorous treatment of delay differential equations dates back to the monograph [24], while, more recently (see $[22,23,25])$, new notions of ad hoc derivatives have been introduced to study the stochastic calculus for path-dependent stochastic differential equations. Since then, such results have been then generalized in several directions; see, for example, [26-32] and the references therein. We would like to mention that the path-dependent calculus has revealed itself since its inception as a powerful tool to model financial markets exhibiting delay and also path-dependent options.

Analogously, in [33-35], the authors proposed an ad hoc notion of viscosity solutions to path-dependent PDE which, similar to the relation established by the FeynmanKac theorem between a stochastic differential equation (SDE) and its deterministic counterpart, relates a path-dependent SDE to a corresponding path-dependent PDE, by exploiting the theory of BSDE, hence by using the notion of nonlinear expectations; see, for example, [36].

Recently, the development of the theory of delayed stochastic differential equations has made one step further to include, besides the delay in the forward SDE, also a delay component in the backward equation. In particular, in [37], the authors proposed a new type of stochastic delay equation, whose generator may depend on the past values of the BSDE itself. As mentioned above, this peculiarity is highly nontrivial, as witnessed by several examples reported in [37], where the authors show how the uniqueness property for the solution fails to be true.

To overcome the latter problem, some additional assumptions have to be taken into account, as in [38], where, exploiting the notion of viscosity solution proposed in [33], the connection between forward-backward SDE with delay both in the forward and in the backward component and a new type of path-dependent PDE has been proved.

In the present paper, we exploit the aforementioned results obtained in [38], to generalize the financial setting developed in $[18,21]$, allowing for path-dependent hedging strategies, that is, plans to reduce the financial risk associated with adverse price movements of, for example, assets in which one has invested, and collateralization scheme with delay. We recall that the collateralization represents the situation in which a borrower pledges an asset as recourse to the lender to hedge the case of the borrower's default. In particular, the collateralization of assets gives, for example, banks a sufficient level of reassurance against the default risk. The latter banking practice allows loans to be issued to individuals or companies which do not belong to the set of the ones having optimal credit history or good debt rating. We underline the notion that our approach is particularly suitable to treat financial frameworks characterized by delays in the default procedure; see, for example, [18, Sec. 3, Sec. 4], the latter being the object of our future works.

Moreover, we underline that analogous approaches can be fruitfully exploited within frameworks characterized by stochastic optimal control problems, as has been made in, for example, [31, 39]; see also the references therein.

The present paper is so structured: in Section 2, we introduce the mathematical setting; in particular, Section 2.1 is devoted to the introduction to the formalism used, whereas in Section 2.2 we state the main theoretical results that will be then applied in Section 3 to the problem of obtaining a portfolio under credit risk and funding issues. Finally, in Section 3.1, we will derive the main path-dependent pricing PDE.

\section{Forward-Backward Delayed Equation and Related Kolmogorov Equation}

In what follows, we introduce the mathematical setting that allows us to derive the delayed pricing equation we are interested in. The aforementioned framework will be first introduced in its complete generality; for example, we will also consider path-dependent coefficients. Moreover, we underline the notion that the main novelty of the present work is represented by the fact that we can also consider a backward equation with delayed generator, together with the associated Feynman-Kac representation theorem. For the sake of completeness, we report here what has been essentially derived in [38]. 
2.1. Pathwise Derivatives, Functional Itô's Formula, Viscosity Solution, and Time Delayed Forward-Backward System. Let us briefly introduce the setting the path-dependent setting, first developed in $[22,23]$, stating the main results; see also $[22,23,38]$, for a rather complete introduction to the path-dependent calculus, [33] which concerns the concept of viscosity solution to path-dependent PDE, and [37] for general results on BSDE with delayed generator.

2.1.1. Pathwise Derivatives and Functional Itô's Formula. In what follows, we define the mathematical framework needed to construct the solutions of the Kolmogorov equation we will treat later; see, for example, [22, 23, 25] for a complete introduction to the related functional Itô calculus. For ease of notation, we will consider $\mathbb{R}$-valued processes, the generalization to the case of $\mathbb{R}^{d}$-valued processes being straightforward.

We will denote $B(t, \widehat{\phi}):=\phi(t)$, while $\mathbb{F}:=\left(\mathscr{F}_{s}\right)_{s \in[0, T]}$ will be the filtration generated by $B$.

Let $\mathscr{D}:=\mathscr{D}([0, T] ; \mathbb{R})$, where $T<+\infty$ is a positive fixed constant, be the set of Càdlàg $\mathbb{R}$-valued functions, that is, right continuous, with finite left-hand limits. On $\mathscr{D}$, we introduce the norm

$$
\|\phi\|_{T}:=\sup _{s \in[0, T]}|\phi(s)|,
$$

with respect to which $\mathscr{D}$ becomes a Banach space. Similarly on $[0, T] \times \mathscr{D}$, we introduce the pseudometric

$$
\begin{aligned}
d\left((t, \phi),\left(t^{\prime}, \phi^{\prime}\right)\right):= & \left|t-t^{\prime}\right| \\
& +\sup _{s \in[0, T]}\left|\phi(s \wedge t)-\phi^{\prime}\left(r \wedge t^{\prime}\right)\right|,
\end{aligned}
$$

with respect to which $[0, T] \times \mathscr{D}$ becomes a complete pseudometric space.

Let $u:[0, T] \times \mathscr{D} \rightarrow \mathbb{R}$ be a given function; then we say that $u$ is vertically differentiable at $(t, \phi) \in[0, T] \times \mathscr{D}$, if there exist

$$
\partial_{x} u(t, \phi):=\lim _{h \rightarrow 0} \frac{u\left(t, \phi+h \mathbb{1}_{[t, T]}\right)-u(t, \phi)}{h} .
$$

The second-order derivative, when it exists, is denoted by $\partial_{x x} u(t, \phi):=\partial_{x}\left(\partial_{x} u\right)$.

Moreover, taking $t \in[0, T]$ and considering a path $\phi \in \mathscr{D}$, we define

$$
\phi_{t}:=\phi(\cdot \wedge t) \in \mathscr{D} .
$$

As it is standard when dealing with delay equations, we will exploit the following notation: for a path $\phi \in \mathscr{D}$, we will denote by $\phi(t)$ the $\mathbb{R}$-value at time $t$ of $\phi$, whereas $\phi_{t}$ denotes the path up to time $t$.

We say that $u$ is horizontally differentiable at $(t, \phi) \in$ $[0, T] \times \mathscr{D}$ if there exist

$$
\partial_{t} u(t, \phi):=\lim _{h \rightarrow 0_{+}} \frac{u\left(t+h, \phi_{t}\right)-u(t, \phi)}{h},
$$

for $t \in[0, T)$ and $\partial_{t} u(T, \phi):=\lim _{t \rightarrow T_{-}} \partial_{t} u(t, \phi)$.
Let $u:[0, T] \times \mathscr{D} \rightarrow \mathbb{R}$ be nonanticipative; we say that $u \in \mathscr{C}([0, T] \times \mathscr{D})$, if $u$ is continuous on $[0, T] \times \mathscr{D}$ under the pseudometric $d$; moreover, we write that $u \in$ $\mathscr{C}_{b}([0, T] \times \mathscr{D})$, if $u \in \mathscr{C}([0, T] \times \mathscr{D})$ and $u$ is bounded on $[0, T] \times \mathscr{D}$. Eventually, we say that $u \in \mathscr{C}_{b}^{1,2}([0, T] \times \mathscr{D})$, if $u \in \mathscr{C}([0, T] \times \mathscr{D})$ and the derivatives $\partial_{x} u, \partial_{x x} u$, and $\partial_{t} u$ exist, and they are both continuous and bounded. Finally, we denote by $\mathscr{D}^{t}:=\mathscr{D}([t, T] ; \mathbb{R})$ the shifted spaces of Càdlàg paths with corresponding metric and pseudometric.

Exploiting previous notations and following [25], we are going to work with processes $u$ defined on $[0, T] \times \mathscr{C} \rightarrow \mathbb{R}$, being $\mathscr{C}:=\mathscr{C}([0, T] ; \mathbb{R})$, namely, the space of continuous paths. It is worth mentioning that all the definitions stated above can be straightforwardly adapted to the case $u:[0, T] \times$ $\mathscr{C} \rightarrow \mathbb{R}$.

For a continuous function $\phi \in \mathscr{C}([-\delta, T] ; \mathbb{R})$, we denote

$$
\phi_{(t)}:=(\phi(t+\theta))_{\theta \in[-\delta, 0]}
$$

$\delta>0$ being a fixed constant.

The following theorem states a functional version of Itô's formula; see, for example, [22, Theorem 4.1].

Theorem 1 (functional Itô's formula). Let $A$ be a 1dimensional Itô process; namely, $A:[0, T] \times \mathscr{C} \rightarrow \mathbb{R}$ is a continuous $\mathbb{R}$-valued semimartingale defined on the probability space $(\mathscr{C}, \mathbb{F}, \mathbb{P})$ which admits the representation

$$
A(t)=A(0)+\int_{0}^{t} b(r) d r+\int_{0}^{t} \sigma(r) d B(r)
$$

$$
\forall t \in[0, T],
$$

for some suitable processes $b$ and $\sigma$.

If $F \in \mathscr{C}_{b}^{1,2}([0, T] \times \mathscr{C})$, then, for any $t \in[0, T)$, the following change of variable formula holds true:

$$
\begin{aligned}
F\left(t, A_{t}\right)= & F\left(0, A_{0}\right)+\int_{0}^{t} \partial_{t} F\left(r, A_{s}\right) d s \\
& +\int_{0}^{t} \partial_{x} F\left(r, A_{s}\right) b(s) d s \\
& +\frac{1}{2} \int_{0}^{t} \sigma^{2}(s) \partial_{x x} F\left(r, A_{s}\right) d s \\
& +\int_{0}^{t} \partial_{x} F\left(r, A_{s}\right) \sigma(s) d W(s) .
\end{aligned}
$$

2.1.2. Viscosity Solution to the Path-Dependent PDE. In what follows, mainly following [38], we introduce the notion of viscosity solution; see also [33].

Let us denote by $\mathscr{T}$ the set of all stopping times $\tau$ such that, for all $t \in[0, T)$, we have that the set $\{\phi \in \mathscr{C}: \tau(\phi)>t\}$ is an open subset of $\left(\mathscr{C},\|\cdot\|_{T}\right)$, while we define by $\mathscr{T}^{t}$ the set of all stopping times $\tau$ such that, for all $s \in[t, T)$, the set $\left\{\phi \in \mathscr{C}^{t}: \tau(\phi)>s\right\}$ is an open subset of $\left(\mathscr{C}^{t},\|\cdot\|_{T}^{t}\right)$. 
Let $L \geq 0$ and $t<T$; then we denote by $\mathcal{U}_{t}^{L}$ the space of $\mathbb{R}$-valued processes $\lambda$ such that $|\lambda| \leq L$; then, we defined a new probability measure $\mathbb{P}^{t, \lambda}$ by $d \mathbb{P}^{t, \lambda}:=M^{t, \lambda}(T) d \mathbb{P}$, where

$$
M^{t, \lambda}(s):=\exp \left(\int_{t}^{s} \lambda(r) d W(r)-\frac{1}{2} \int_{t}^{s}|\lambda(r)|^{2} d r\right),
$$

P-a.s.

Let us consider the process $\left(X^{t, \phi}(s)\right)_{s \in[0, T]}$ which evolves according to

$$
\begin{aligned}
X^{t, \phi}(s)= & \phi(t)+\int_{t}^{s} b\left(r, X^{t, \phi}\right) d r \\
& +\int_{t}^{s} \sigma\left(r, X^{t, \phi}\right) d W(r), \quad s \in[t, T], \\
X^{t, \phi}(s)= & \phi(s), \quad s \in[0, t),
\end{aligned}
$$

where $(t, \phi) \in[0, T] \times \mathscr{C}$ is given, $W$ is standard Brownian motion, and $b$ and $\sigma$ are some suitable coefficients that will be soon better specified. Further, we denote by $\mathbb{G}=\left(\mathscr{G}_{s}\right)_{s \in[0, T]}$ the natural filtration generated by $W$.

Taking into account what we have introduced so far, we are in position to state the main object of analysis in the present work, namely, the following functional pathdependent PDE:

$$
\begin{aligned}
& -\partial_{t} u(t, \phi)-\mathscr{L} u(t, \phi) \\
& \quad-f\left(t, \phi, u(t, \phi), \partial_{x} u(t, \phi) \sigma(t, \phi),(u(\cdot, \phi))_{t}\right) \\
& \quad=0, \\
& u(T, \phi)=h(\phi),
\end{aligned}
$$

for $t \in[0, T), \phi \in \mathscr{C}$. Moreover, indicating by $\delta>0$ a given fixed delay, we have set $(u(\cdot, \phi))_{t}:=(u(t+\theta, \phi))_{\theta \in[-\delta, 0]}$, while, in (11), we have denoted by $\mathscr{L}$ the second-order differential operator given by

$$
\begin{aligned}
\mathscr{L} u(t, \phi):= & \frac{1}{2} \sigma^{2}(t, \phi) \partial_{x x} u(t, \phi) \\
& +b(t, \phi) \partial_{x} u(t, \phi),
\end{aligned}
$$

with $b:[0, T] \times \mathscr{C} \rightarrow \mathbb{R}$ and $\sigma:[0, T] \times \mathscr{C} \rightarrow \mathbb{R}$ being two nonanticipative functionals.

We now introduce the space of the test functions:

$$
\begin{aligned}
& \underline{\mathscr{A}}^{L} u(t, \phi):=\left\{\varphi \in \mathscr{C}_{b}^{1,2}([0, T] \times \mathscr{C}): \exists \tau_{0}\right. \\
& \in \mathscr{T}_{+}^{t}, \varphi(t, \phi)-u(t, \phi) \\
& \left.=\min _{\tau \in \mathscr{T}^{t}} \mathscr{E}_{t}^{L}\left[(\varphi-u)\left(\tau \wedge \tau_{0}, X^{t, \phi}\right)\right]\right\}, \\
& \overline{\mathscr{A}}^{L} u(t, \phi):=\left\{\varphi \in \mathscr{C}_{b}^{1,2}([0, T] \times \mathscr{C}): \exists \tau_{0}\right. \\
& \in \mathscr{T}_{+}^{t}, \varphi(t, \phi)-u(t, \phi) \\
& \left.=\max _{\tau \in \mathscr{T}^{t}} \overline{\mathscr{E}}_{t}^{L}\left[(\varphi-u)\left(\tau \wedge \tau_{0}, X^{t, \phi}\right)\right]\right\},
\end{aligned}
$$

where $\mathscr{T}_{+}^{t}:=\left\{\tau \in \mathscr{T}^{t}: \tau>t\right\}$, if $t<T$ and $\mathscr{T}_{+}^{T}:=\{T\}$. Also, for any $\xi \in L^{2}\left(\mathscr{F}_{T}^{t} ; \mathbb{P}\right), \underline{\mathscr{E}}_{t}^{L}(\xi):=\inf _{\lambda \in \mathscr{U}_{t}^{L}} \mathbb{E}^{\mathbb{P}^{t, \lambda}}(\xi)$ and $\overline{\mathscr{E}}_{t}^{L}(\xi):=$ $\sup _{\lambda \in \mathcal{U}_{t}^{L}} \mathbb{E}^{\mathbb{P} t, \lambda}(\xi)$ are nonlinear expectations.

The definition of a viscosity solution to the functional PDE (11) (see, e.g., [33, Def. 3.3] or [38, Def. 2]) reads as follows.

Definition 2. Let $u \in \mathscr{C}_{b}([0, T] \times \mathscr{C})$ such that $u(T, \phi)=h(\phi)$, for all $\phi \in \mathscr{C}$.

(a) For any $L \geq 0$, one says that $u$ is a viscosity $L$ subsolution of (11) if at any point $(t, \phi) \in[0, T] \times \mathscr{C}$, and, for any $\varphi \in \underline{\mathscr{A}}^{L} u(t, \phi)$, it holds that

$$
\begin{aligned}
-\partial_{t} \varphi & (t, \phi)-\mathscr{L} \varphi(t, \phi) \\
& -f\left(t, \phi, \varphi(t, \phi), \partial_{x} \varphi(t, \phi) \sigma(t, \phi),(\varphi(\cdot, \phi))_{t}\right)
\end{aligned}
$$

$\leq 0$.

(b) For any $L \geq 0$, one says that $u$ is a viscosity $L$ supersolution of (11) if at any point $(t, \phi) \in[0, T] \times \mathscr{C}$, and, for any $\varphi \in \overline{\mathscr{A}}^{L} u(t, \phi)$, one has

$$
\begin{aligned}
-\partial_{t} \varphi & (t, \phi)-\mathscr{L} \varphi(t, \phi) \\
& -f\left(t, \phi, \varphi(t, \phi), \partial_{x} \varphi(t, \phi) \sigma(t, \phi),(\varphi(\cdot, \phi))_{t}\right) \\
& \geq 0 .
\end{aligned}
$$

(c) One says that $u$ is a viscosity subsolution, respectively, supersolution, of (11), if $u$ is a viscosity $L$-subsolution, respectively, $L$-supersolution, of (11), for some $L \geq 0$.

(d) One says that $u$ is a viscosity solution of (11) if $u$ is both a viscosity subsolution and supersolution of (11).

2.1.3. Time Delayed Forward-Backward System. The present section is devoted to the characterization of the delayed forward-backward system we are interested in. In particular, we will look for a triplet,

$$
\left(X^{u, \phi}, Y^{u, \phi}, Z^{u, \phi}\right)_{(u, \phi) \in[0, T] \times \mathscr{C}},
$$

of stochastic processes such that the following decoupled forward-backward system holds:

$$
\begin{aligned}
X^{u, \phi}(t)= & \phi(u)+\int_{u}^{t} b\left(s, X^{u, \phi}\right) d s \\
& +\int_{u}^{t} \sigma\left(s, X^{u, \phi}\right) d W(s), \quad t \in[u, T], \\
X^{u, \phi}(t)= & \phi(t), \quad t \in[0, s), \\
Y^{u, \phi}(t)= & h\left(X^{u, \phi}\right) \\
& +\int_{t}^{T} f\left(s, X^{u, \phi}, Y^{u, \phi}(s), Z^{u, \phi}(s), Y_{s}^{u, \phi}\right) d s \\
& -\int_{t}^{T} Z^{u, \phi}(s) d W(s), \quad t \in[u, T],
\end{aligned}
$$




$$
\begin{aligned}
& Y^{u, \phi}(t)=Y^{t, \phi}(t), \\
& Z^{u, \phi}(t)=0,
\end{aligned}
$$$$
t \in[0, u)
$$

Let us first focus on the forward component $X$ appearing in system (17), also assuming the following.

Assumptions 3. Let us consider two nonanticipative functionals $b:[0, T] \times \mathscr{C} \rightarrow \mathbb{R}$ and $\sigma:[0, T] \times \mathscr{C} \rightarrow \mathbb{R}$ such that

$\left(\mathrm{A}_{1}\right) b$ and $\sigma$ are continuous;

$\left(\mathrm{A}_{2}\right)$ there exists $\ell>0$ such that, for any $t \in[0, T], \phi, \phi^{\prime} \in$ $\mathscr{C}$,

$$
\begin{aligned}
& \left|b(t, \phi)-b\left(t, \phi^{\prime}\right)\right|+\left|\sigma(t, \phi)-\sigma\left(t, \phi^{\prime}\right)\right| \\
& \quad \leq \ell\left\|\phi-\phi^{\prime}\right\|_{T} .
\end{aligned}
$$

In light of Assumptions 3, the following theorem states both the existence and the uniqueness for the process $\left(X^{u, \phi}(t)\right)_{t \in[0, T]}$; see, for example, [38, Th. 4].

Theorem 4. Let the coefficients $b$ and $\sigma$ satisfy Assumptions $3\left(A_{1}\right)-\left(A_{2}\right)$. Let $(t, \phi),\left(t^{\prime}, \phi^{\prime}\right) \in[0, T] \times \mathscr{C}$ be given. Then, there exists a unique continuous and adapted stochastic process $\left(X^{u, \phi}(t)\right)_{t \in[0, T]}$, such that

$$
\begin{aligned}
X^{u, \phi}(t)= & \phi(u)+\int_{u}^{t} b\left(s, X^{t, \phi}\right) d s \\
& +\int_{u}^{t} \sigma\left(s, X^{u, \phi}\right) d W(s), \quad t \in[u, T], \\
X^{u, \phi}(t)= & \phi(t), \quad t \in[0, u) .
\end{aligned}
$$

In order to analyse the delayed backward SDE appearing in (17), let us first introduce the main reference spaces we will work with.

Definition 5. (i) Let $\mathscr{H}_{t}^{2}$ denote the space of $\mathbb{G}^{u}$-progressively measurable processes $Z: \Omega \times[u, T] \rightarrow \mathbb{R}$, such that

$$
\mathbb{E}\left[\int_{u}^{T}|Z(s)|^{2} d s\right]<\infty .
$$

(ii) Let $\mathcal{S}_{u}^{2}$ be the space of continuous $\mathbb{G}^{u}$-progressively measurable processes $Y: \Omega \times[u, T] \rightarrow \mathbb{R}$ such that

$$
\mathbb{E}\left[\sup _{u \leq s \leq T}|Y(s)|^{2}\right]<\infty .
$$

Moreover, we will equip the spaces $\mathscr{H}_{u}^{2}$ and $\mathcal{S}_{u}^{2}$ with the following norms:

$$
\begin{aligned}
\|Z\|_{\mathscr{H}_{u}^{2}}^{2} & =\mathbb{E}\left[\int_{u}^{T} e^{\beta s}|Z(s)|^{2} d s\right], \\
\|Y\|_{\delta_{u}^{2}}^{2} & =\mathbb{E}\left[\sup _{u \leq s \leq T} e^{\beta s}|Y(s)|^{2}\right],
\end{aligned}
$$

where $\beta>0$ is a given constant.
Further, in order to deal with the delayed backward SDE appearing in (17), we will assume the following.

Assumptions 6. Let

$$
\begin{array}{r}
f:[0, T] \times \mathscr{C} \times \mathbb{R} \times \mathbb{R} \times \mathscr{C}([-\delta, 0] ; \mathbb{R}) \longrightarrow \mathbb{R}, \\
h: \mathscr{C} \longrightarrow \mathbb{R},
\end{array}
$$

such that the following hold:

$\left(\mathrm{B}_{1}\right)$ There exist $L, K, M>0, p \geq 1$ and a probability measure $\alpha$ on $([-\delta, 0], \mathscr{B}([-\delta, 0]))$ such that, for any $t \in[0, T], \phi \in \mathscr{C},(y, z),\left(y^{\prime}, z^{\prime}\right) \in \mathbb{R} \times \mathbb{R}, \hat{y}, \hat{y}^{\prime} \in$ $\mathscr{C}([-\delta, 0] ; \mathbb{R})$, we have the following:

(i) $\phi \longmapsto f(t, \phi, y, z, \widehat{y})$ is continuous,

(ii) $\left|f(t, \phi, y, z, \hat{y})-f\left(t, \phi, y^{\prime}, z^{\prime}, \widehat{y}\right)\right|$

$$
\leq L\left(\left|y-y^{\prime}\right|+\left|z-z^{\prime}\right|\right)
$$

(iii) $\left|f(t, \phi, y, z, \widehat{y})-f\left(t, \phi, y, z, \hat{y}^{\prime}\right)\right|^{2}$

$$
\leq K \int_{-\delta}^{0}\left|\widehat{y}(\theta)-\widehat{y}^{\prime}(\theta)\right|^{2} \alpha(d \theta),
$$

(iv) $|f(t, \phi, 0,0,0)|<M\left(1+\|\phi\|_{T}^{p}\right)$.

$\left(\mathrm{B}_{2}\right)$ The function $f(\cdot, \cdot, y, z, \widehat{y})$ is $\mathbb{F}$-progressively measurable, for any $(y, z, \widehat{y}) \in \mathbb{R} \times \mathbb{R} \times \mathscr{C}([-\delta, 0] ; \mathbb{R})$.

$\left(\mathrm{B}_{3}\right)$ The function $h$ is continuous and, for all $\phi \in \mathscr{C}$,

$$
|h(\phi)| \leq M\left(1+\|\phi\|_{T}^{p}\right) .
$$

Hence, we are in position to report the result which states both the existence and the uniqueness for the solution to the BSDE with delayed generator we are considering; see, for example, [38, Th. 7].

Theorem 7. If Assumptions $6\left(B_{1}\right)-\left(B_{3}\right)$ hold true and $K$ and $\delta$ are small enough, namely, if there exists a constant $\gamma \in(0,1)$ such that

$$
K \frac{\gamma e^{\left(\gamma+6 L^{2} / \gamma\right) \delta}}{(1-\gamma) L^{2}} \max \{1, T\}<\frac{1}{290}
$$

then there exists a unique solution $\left(Y^{u, \phi}, Z^{u, \phi}\right)_{(u, \phi) \in[0, T] \times \mathscr{C}}$ to the backward stochastic differential system (17), such that

$$
\left(Y^{u, \phi}, Z^{u, \phi}\right) \in \mathcal{S}_{u}^{2} \times \mathscr{H}_{u}^{2},
$$

for all $u \in[0, T]$; moreover, $u \mapsto\left(Y^{u, \phi}, Z^{u, \phi}\right)$ is continuous from $[0, T]$ into $\mathcal{S}_{0}^{2} \times \mathscr{H}_{0}^{2}$.

2.2. Viscosity Solution for the Path-Dependent PDE. The present subsection is mainly based on [38] and it is devoted to the study of viscosity solutions to the path-dependent equation (11). In what follows, we will consider that Assumptions $6\left(B_{1}\right)-\left(B_{3}\right)$ hold true. 
It is worth mentioning that typical generators $f$ satisfying Assumptions 6 are of the following form:

$$
\begin{aligned}
& f_{1}(t, \phi, y, z, \widehat{y}):=K \int_{-\delta}^{0} \widehat{y}(s) d s, \\
& f_{2}(t, \phi, y, z, \hat{y}):=K \hat{y}(t-\delta) .
\end{aligned}
$$

Remark 8. If $g:[0, T] \rightarrow \mathbb{R}$ is a measurable and bounded function with $g(t)=0$ for $t<0$, the linear time delayed generator

$$
f(t, \phi, y, z, \widehat{y})=\int_{-\delta}^{0} g(t+\theta) \hat{y}(\theta) \alpha(d \theta)
$$

satisfies Assumptions 6.

Let us define the function $u:[0, T] \times \mathscr{C} \rightarrow \mathbb{R}$ by

$$
u(t, \phi):=Y^{t, \phi}(t), \quad(t, \phi) \in[0, T] \times \mathscr{C}
$$

notice that $u(t, \phi)$ is deterministic; in fact, $Y^{t, \phi}(t)$ is $\mathscr{G}_{t}^{t} \equiv \mathcal{N}$ measurable. Thus, we have the following results which are the Feynman-Kac type result adapted to the current pathdependent setting.

Theorem 9 (existence). Let Assumptions 3 and 6 hold true; then, if the delay $\delta$ and the Lipschitz constant $K$ are sufficiently small, namely, if condition (26) is verified, then the pathdependent PDE (11) admits at least one viscosity solution.

Proof. See, for example, [38, Th. 11].

Theorem 10. Let Assumptions 3 and 6 hold true as well as condition (26). Then, there exists a continuous nonanticipative functional $u:[0, T] \times \mathscr{C} \rightarrow \mathbb{R}$ such that

$$
u\left(t, X^{u, \phi}\right)=Y^{u, \phi}(t), \quad \forall t \in[0, T], \text { a.s. },
$$

for any $(u, \phi) \in[0, T] \times \mathscr{C}$, which is a viscosity solution to the path-dependent PDE (11).

Proof. See, for example, [38, Th. 14].

\section{Pricing under Counterparty Risk}

In the present section, we are going to apply previously derived results to the pricing of financial derivatives under counterparty risk and funding issues. In order to derive the pricing equation, we closely follow [21, Sec. 2]; see also, for example, $[10,40]$ and the references therein.

Let us consider a standard filtered probability space $\left(\Omega, \mathscr{G},\left(\mathscr{G}_{s}\right)_{s \in[0, T]}, \mathbb{Q}\right)$, with $T<+\infty$ being a fixed positive constant, while the filtration $\mathscr{G}_{s}$ represents all the information available on the market at a given time $s$. Our goal is to derive a portfolio of financial contracts between two parties, namely, the investor, which will be denoted by $I$, and the counterparty, which will be denoted by $C$.

In order to work in a realistic and concrete financial framework, we include the risk of default. In particular, we denote by $\tau_{I}$ and $\tau_{C}$ the $\mathscr{G}$-measurable stopping time representing the default time of $I$ and $C$, respectively. Moreover, we prescribe that $\tau_{I}$ and $\tau_{C}$ have intensity $\lambda_{I}>0$ and $\lambda_{C}>0$, respectively, and are indicated by $\tau:=\tau_{I} \wedge \tau_{C}$ and by $\lambda:=$ $\lambda_{I}+\lambda_{C}$. Recalling that a risk-neutral measure $\mathbb{Q}$ is nothing but a probability measure such that each share price equals the discounted expectation of the share price under $\mathbb{Q}$ itself, in what follows, $\left(\mathscr{F}_{s}\right)_{s \in[0, T]}$ indicates the default-free filtration generated by the asset $S$ which evolves under the risk-neutral measure $\mathbb{Q}$ and according to the following SDE:

$$
\begin{aligned}
\frac{d S(t)}{S(t)} & =r_{t} d t+\sigma\left(t, S_{t}\right) d W(t), \\
S_{0} & =s_{0}>0 \in \mathbb{R}_{+},
\end{aligned}
$$

where $r$ is $\mathscr{F}$-measurable process indicating the risk-free rate. We also assume that there exists a risk-free account $B$ whose dynamic is given by

$$
\begin{aligned}
d B(t) & =r_{t} B(t) d t \\
B_{0} & =1
\end{aligned}
$$

where we have used the notation introduced in Section 2, while the assumptions on the coefficients appearing in (32)(33) will be specified in a while. We underline the notion that we indicate by $S(t)$ the present $\mathbb{R}$-value of the process $S$, whereas $S_{t}$ denotes the whole path up to time $t$, so that, in complete generality, we have assumed both the risky asset and the riskless rate to be path-dependent.

Remark 11. Until now, we have worked under the strong assumption that there exists a risk-free rate $r$ with a corresponding risk-free account. Nevertheless, the latter assumption turns out to be rather unrealistic in concrete financial markets, and this enlightens a major strength of our approach, since it allows deriving a portfolio that is independent of the risk-free rate $r$.

Given a rate $\xi(s)$, we will denote the discount factor associated with $\xi$ by

$$
D(u, t ; \xi):=e^{-\int_{u}^{t} \xi_{s} d s},
$$

and we also define $D(u, t):=D(u, t ; r)$.

Following [21, Sec. 2.1], we construct a replicating portfolio taking all future cash flows and then discounting them at the risk-free rate $r$. Moreover, to treat the problem in its full generality, we will assume the following processes to be possibly path-dependent, hence stating a difference with respect to what has been done in [21].

In particular, we have first to consider the payments due to the contract itself, which is a predictable process $\pi_{t}$ and the terminal payoff of the claim $\Phi\left(S_{T}\right)$, so that, at time $t$, the cumulated discounted flow is given by

$$
\mathbb{1}_{\{\tau>T\}} D(0, T) \Phi\left(S_{T}\right)+\int_{t}^{\tau} D(t, s) \pi_{s} d s .
$$


We also have a random variable $\theta_{\tau}$ representing the cash flow due to the default of one of the parties; hence, the resulting cash flow is given by

$$
\mathbb{1}_{\{t<\tau<T\}} D(t, \tau) \theta_{\tau}=\mathbb{1}_{\{t<\tau<T\}} \int_{t}^{T} D(t, s) \theta_{s} d \mathbb{1}_{\{\tau \leq s\}} .
$$

We consider further a collateral account $C_{t}$, namely, an asset or some other financial good that a borrower offers to a lender to secure a loan, and we will use the convention that $C_{t}>0$ and $C_{t}<0$ for the investor being the collateral taker and the collateral provider, respectively. Moreover, we assume that the collateral account is subjected to an interest rate $c_{t}$, which might be different according to what party the collateral taker is in; namely, we have

$$
c_{t}=\mathbb{1}_{\left\{C_{t}>0\right\}} c_{t}^{+}+\mathbb{1}_{\left\{C_{t}<0\right\}} c_{t}^{-} .
$$

Allowing also for rehypothecation, namely, allowing banks and brokers to use assets that have been posted as collateral by their clients, we end up with the following cash flow:

$$
\int_{t}^{\tau} D(t, s)\left(r_{s}-c_{s}\right) C_{s} d s
$$

The contract will be hedged by a cash position denoted by $H_{t}$, respectively, by exploiting a position in a risky asset, denoted by $F_{t}$. As we have already done, we use the convention that $F_{t}>0$ and $F_{t}<0$ if the investor is borrowing money and if he is investing money, respectively. Again, we assume the existence of two different rates

$$
f_{t}=\mathbb{1}_{\left\{F_{t}>0\right\}} f_{t}^{+}+\mathbb{1}_{\left\{F_{t}<0\right\}} f_{t}^{-},
$$

so that the funding component cash flow reads as follows:

$$
\int_{t}^{\tau} D(t, s)\left(r_{s}-f_{s}\right) F_{s} d s
$$

A similar convention holds for $H_{t}$, so that (39)-(40) become

$$
\begin{gathered}
h_{t}=\mathbb{1}_{\left\{H_{t}>0\right\}} h_{t}^{+}+\mathbb{1}_{\left\{H_{t}<0\right\}} h_{t}^{-}, \\
\int_{t}^{\tau} D(t, s)\left(h_{s}-f_{s}\right) H_{s} d s .
\end{gathered}
$$

Eventually, summing up all the aforementioned cash flows (35), (36), (38), (40), and (42), we have that the value of the portfolio $V$ is given by

$$
\begin{aligned}
& V(t)=\mathbb{E}_{t}^{\mathscr{G}}\left[\int_{t}^{\tau} D(t, s)\right. \\
& \left.\cdot\left(\pi_{s}+\left(r_{s}-c_{s}\right) C_{s}+\left(r_{s}-f_{s}\right) F_{s}-\left(f_{s}-h_{s}\right) H_{s}\right)\right] \\
& +\mathbb{E}_{t}^{\mathscr{G}}\left[\mathbb{1}_{\{\tau>T\}} D(t, T) \Phi\left(S_{T}\right)+\mathbb{1}_{\{t<\tau<T\}} D(t, \tau) \theta_{\tau}\right],
\end{aligned}
$$

where we have denoted $\mathbb{E}_{t}^{\mathscr{G}}[\cdot]:=\mathbb{E}\left[\cdot \mid \mathscr{G}_{t}\right]$. Further, since $V(s)=F_{s}+H_{s}+C_{s}$, we can substitute it in (43), obtaining

$$
\begin{aligned}
& V(t)=\mathbb{E}_{t}^{\mathscr{G}}\left[\int _ { t } ^ { \tau } D ( t , s ) \left(\pi_{s}+\left(f_{s}-c_{s}\right) C_{s}\right.\right. \\
& \left.\left.+\left(r_{s}-f_{s}\right) V(s)-\left(r_{s}-h_{s}\right) H_{s}\right)\right] \\
& \quad+\mathbb{E}_{t}^{\mathscr{G}}\left[\mathbb{1}_{\{\tau>T\}} D(t, T) \Phi\left(S_{T}\right)+\mathbb{1}_{\{t<\tau<T\}} D(t, \tau) \theta_{\tau}\right]
\end{aligned}
$$

For a concrete example of how (44) is practically derived, we refer to [21, Sec. 2.2].

Switching to the default-free filtration, we can exploit the results stated in [41, Sec. 5.1] and [42, Lemma 3.8.1]; see also [21, Lemma 3.1, Lemma 3.3]. In particular, let us recall that $\mathscr{G}$ is the standard filtration, whereas $\mathscr{F}$ denotes the default-free filtration.

Lemma 12. For any $X \mathscr{G}$-measurable random variable and any $t \geq 0$, it holds that

$$
\mathbb{E}_{t}^{\mathscr{G}}\left[\mathbb{1}_{\{t<\tau \leq s\}} X\right]=\mathbb{1}_{\{\tau>t\}} \frac{\mathbb{E}_{t}^{\mathscr{F}}\left[\mathbb{1}_{\{t<\tau \leq s\}} X\right]}{\mathbb{E}_{t}^{\mathscr{F}}\left[\mathbb{1}_{\{\tau>t\}}\right]} .
$$

In particular, we have that, for any $\mathscr{G}_{t}$-measurable random variable $Y$, there exists an $\mathscr{F}_{t}$-measurable random variable $Z$ such that

$$
\mathbb{1}_{\{\tau>t\}} X=\mathbb{1}_{\{\tau>t\}} Z
$$

Lemma 13. Let $\varphi_{s}$ be a predictable process and let $\tau_{I}$ and $\tau_{C}$ be a stopping time with intensity $\lambda_{t}^{I}>0$ and $\lambda_{t}^{C}>0$, respectively. Assuming that $\tau_{I}$ and $\tau_{C}$ are independent and denoting $\tau:=$ $\tau_{I} \wedge \tau_{C}$ and $\lambda_{t}=\lambda_{t}^{I}+\lambda_{t}^{C}$, then one has

$$
\begin{aligned}
\mathbb{E}_{t}^{\mathscr{G}} & {\left[\mathbb{1}_{\{t<\tau<T\}} \mathbb{1}_{\left\{\tau_{I}<\tau_{C}\right\}} \varphi_{\tau}\right] } \\
& =\mathbb{1}_{\{\tau>t\}} \mathbb{E}_{t}^{\mathscr{F}}\left[\int_{t}^{T} D(t, s ; \lambda) \lambda_{t}^{I} \varphi_{s} d s\right] .
\end{aligned}
$$

So, by an application of Lemmas 12 and 13 and with slight abuse of notation, we denote again by $V(t)$ the portfolio evaluated under the default-free filtration $\mathscr{F}$; namely,

$$
\begin{aligned}
& V(t)=\mathbb{E}_{t}^{\mathscr{F}}\left[\int _ { t } ^ { \tau } D ( t , s ; r + \lambda ) \left(\pi_{s}+\left(f_{s}-c_{s}\right) C_{s}\right.\right. \\
& \left.\left.+\left(r_{s}-f_{s}\right) V(t)-\left(r_{s}-h_{s}\right) H_{s}\right)\right]+\mathbb{E}_{t}^{\mathscr{F}}[D(t, T ; r \\
& \left.\quad+\lambda) \Phi\left(S_{T}\right)+D(t, \tau ; r+\lambda) \theta_{\tau}\right] .
\end{aligned}
$$

By (48) and proceeding as in [21, Sec. 3], we can immediately obtain the BSDE formulation for the portfolio $V$; that is, we have that $V$ evolves according to

$$
\begin{aligned}
& d V(s)=-\left(\pi_{s}+\left(f_{s}-c_{s}\right) C_{s}-\left(\lambda_{s}+f_{s}\right) V(s)\right. \\
& \left.\quad-\left(r_{s}-h_{s}\right) H_{s}\right) d s-Z(s) d W(s), \\
& V(T)=\Phi\left(S_{T}\right) .
\end{aligned}
$$


3.1. The Pricing Path-Dependent PDE. In the previous section, we have derived the BSDE that describes the evolution of the financial portfolio, while, in the present section, we are going to better specify the mathematical assumptions regarding (49). We would like to underline the notion that while some of the following assumptions are mainly taken from [21, Sec. 4], some others are here formalized, to our best knowledge, for the first time.

We assume that the dividend process $\pi$ depends on time $t$ and on the underlying $S$; moreover, we assume the dependence to be possibly path-dependent; namely, we have $\pi\left(t, S_{t}\right)$. Further, we assume that $\pi$ satisfies Assumptions 3.

In what follows, all the rates $r, f, c, h$, and $\lambda$ are taken to be deterministic and bounded in time and possibly dependent on past values.

We assume that $\theta$ has the form

$$
\begin{aligned}
\theta_{t}= & \epsilon(t)-\mathbb{1}\left\{\tau_{C}<\tau_{I}\right\} \operatorname{LGD}_{C}\left(\epsilon(t)-C_{t}\right)^{+} \\
& +\mathbb{1}\left\{\tau_{I}<\tau_{C}\right\} \operatorname{LGD}_{I}\left(\epsilon(t)-C_{t}\right)^{-}
\end{aligned}
$$

where LGD denotes the loss given default, commonly defined as the share of an asset that is lost when a borrower defaults, and $\left(\epsilon(t)-C_{t}\right)^{+}$and $\left(\epsilon(t)-C_{t}\right)^{-}$denote the positive part and the negative part, respectively. We will not enter here financial details regarding $\theta_{t}$, since it would go beyond the aim of the present work, but we refer the interested reader to [10] for a deep treatment of closeout values. We assume then that $\epsilon(t)=$ $V(t)$.

The hedging term $H$ is of the form $H_{s}=H\left(s, S_{s}, V(s)\right.$, $Z(s))$ and it satisfies Assumptions 6; moreover, the diffusion term $\sigma$, appearing in (32), satisfies Assumptions 3.

Last but not least, since this is the main novelty of the present approach, we assume that the collateral depends on portfolio past values. As said above, this implies that the BSDE is highly irregular, and even the existence and uniqueness of a solution are in general not granted under standard assumptions. However, as pointed out in [15, Rem. $5.5]$, in practice, it often happens that the collateralization scheme is path-dependent in $V$. We thus assume that the collateral $C$ is of the following particular form:

$$
\begin{aligned}
& C_{(s)}=\alpha_{t} \bar{V}_{(t)}, \\
& \bar{V}_{(t)}:=\frac{1}{\delta} \int_{-\delta}^{0} V(t+s) d s,
\end{aligned}
$$

where $\alpha_{t} \in(0,1]$; that is, we assume the collateral to be a fraction of a time average of the portfolio. Notice that in principle also $\theta$ in (50) depends on $\bar{V}_{(t)}$ so that in what follows we will use the notation $\theta_{s}\left(\bar{V}_{(s)}\right)$. Moreover, in order to satisfy condition (26), we have that $\delta$ is positive and small enough. Alternatively, we can set $\delta=T$, at the cost of assuming that the Lipschitz constant in (32) satisfies condition (26).

We would like to underline the notion that previous choice is just one of the possible schemes admitted in our mathematical setting; see, for example, (28)-(29).

The aforementioned assumptions can be better formalized as follows.
Assumptions 14. One has the following:

(C1) The parameters $r, f, c, h$, and $\lambda$ are all bounded elements of $\mathscr{C}$.

(C2) $H:[0, T] \times \mathscr{C} \times \mathbb{R} \times \mathbb{R} \rightarrow \mathbb{R}$, and there exist positive constants $L_{H}>0$ and $M_{H}>0$ and $p \geq 1$ such that

(i) $\phi \longmapsto H(t, \phi, y, z)$ is continuous,

(ii) $\left|H(t, \phi, y, z)-H\left(t, \phi, y^{\prime}, z^{\prime}\right)\right|$

$$
\leq L_{H}\left(\left|y-y^{\prime}\right|+\left|z-z^{\prime}\right|\right),
$$

(iii) $|H(t, \phi, 0,0)|<M_{H}\left(1+\|\phi\|_{T}^{p}\right)$.

(C3) $\Phi: \mathscr{C} \rightarrow \mathbb{R}$ is continuous and, for all $\phi \in \mathscr{C}$, there exist $M_{\Phi}>0$ and $p \geq 1$, such that

$$
|\Phi(\phi)| \leq M_{\Phi}\left(1+\|\phi\|_{T}^{p}\right) .
$$

(C4) $\sigma:[0, T] \times \mathscr{C} \rightarrow \mathbb{R}$ is continuous and, for any $t \in$ $[0, T], \phi, \phi^{\prime} \in \mathscr{C}$, there exists $\ell_{\sigma}>0$ such that

$$
\left|\sigma(t, \phi)-\sigma\left(t, \phi^{\prime}\right)\right| \leq \ell_{\sigma}\left\|\phi-\phi^{\prime}\right\|_{T} .
$$

In light of Assumptions 14, let us consider the following forward-backward collateralization scheme:

$$
\begin{aligned}
S^{u, \phi}(t)= & \phi(t)+\int_{u}^{t} r_{s} d s+\int_{u}^{t} \sigma\left(s, S_{s}^{u, \phi}\right) d W(s), \\
s \in[u, T], & \\
S^{u, \phi}(t)= & \phi(t), \quad t \in[0, u), \\
V^{u, \phi}(t)= & \Phi\left(S_{T}^{u, \phi}\right) \\
& +\int_{t}^{T} B\left(s, S_{s}^{u, \phi}, V^{u, \phi}(s), Z^{u, \phi}(s), V_{(s)}^{u, \phi}\right) d s \\
& -\int_{t}^{T} Z^{u, \phi}(s) d W(s), \\
V^{u, \phi}(t)= & V^{t, \phi}(t), \quad t \in[0, u),
\end{aligned}
$$

with

$$
\begin{aligned}
B:= & \left(f_{s}+\lambda_{s}\right) V^{u, \phi}(s)-\pi_{s}-\theta_{s}\left(\bar{V}_{(s)}\right) \\
& +\left(r_{s}-h_{s}\right) H\left(s, S_{s}, V(s), Z(s)\right) \\
& -\alpha_{s}\left(f_{s}-c_{s}\right) \bar{V}_{(s)} .
\end{aligned}
$$

Remark 15. It is worth mentioning that, in the setting represented by (55), we are generalizing the framework considered in [21]. In fact, we have that, besides assuming the collateralization scheme to be dependent on the past values of $V$, we are also considering both the hedging strategy and the terminal payoff to be possibly path-dependent. Moreover, we do not require any differentiability assumption on $\Phi$. The latter is a crucial point when dealing with concrete financial derivatives often characterized by terminal payoffs which are Lipschitz continuous but fail to be differentiable. 
With respect to the forward-backward system (55), we have the following.

Theorem 16. Let one consider the forward-backward delayed system (55) with Assumptions 14. Then, for every $(u, \phi) \in$ $[0, T] \times \mathscr{C}$, it holds that

$$
V^{u, \phi}(t)=u\left(t, S^{u, \phi}\right), \quad \forall t \in[u, T],
$$

where $u(t, \phi)=V^{t, \phi}(t)$ is a viscosity solution of the following path-dependent PDE:

$$
\begin{aligned}
& \partial_{t} u(t, \phi)+\frac{1}{2} \sigma^{2}(t, \phi) \partial_{x x}^{2} u(t, \phi)+r_{t} \partial_{x} u(t, \phi) \\
& =B\left(t, \phi, u(t, \phi), \partial_{x} u(t, \phi) \sigma(t, \phi),(u(\cdot, \phi))_{t}\right), \\
& \quad \phi \in \mathscr{C}, t \in[0, T), \\
& u(T, \phi)=\Phi(\phi), \quad \phi \in \mathscr{C},
\end{aligned}
$$

with

$$
\begin{aligned}
B(t, \phi, y, z, \widehat{y}):= & \left(f_{s}+\lambda_{s}\right) y-\pi_{s}-\theta_{s}(\widehat{y}) \\
& +\left(r_{s}-h_{s}\right) H(s, \phi, y, z) \\
& -\alpha_{s}\left(f_{s}-c_{s}\right) \hat{y} .
\end{aligned}
$$

Proof. Because of Assumptions 14, we have that (55) satisfies Assumptions 3 and 6; hence, exploiting Theorems 4 and 7, we have existence and uniqueness of a solution to the delayed forward-backward system (55). Thus, by using the results stated in the previous sections, we can derive the characterization of (55) given in (65). In particular, we obtain that Theorem 10 holds true, and the claim follows.

We would like to underline the notion that the scheme described by (55) still depends on the nonrealistic assumption of a risk-free rater. Then, in order to consider a more concrete case, namely, without the risk-free rate, we will exploit the results stated in [21, Sec. 6]. In particular, we will assume the hedging strategy to be a classical delta-hedging strategy; namely,

$$
H\left(s, S_{s}, V(s), Z(s)\right):=S_{t} \frac{Z(t)}{\sigma\left(t, S_{t}\right)},
$$

so that (55) can be rewritten as follows:

$$
\begin{aligned}
& S^{u, \phi}(t)=\phi(t)+\int_{u}^{t} h_{s} d s+\int_{u}^{t} \sigma\left(s, S_{s}^{u, \phi}\right) d W(s), \\
& s \in[u, T], \\
& S^{u, \phi}(t)=\phi(t), \quad t \in[0, u), \\
& V^{u, \phi}(t)=\Phi\left(S_{T}^{u, \phi}\right)+\int_{t}^{T} \widetilde{B}\left(s, S_{s}^{u, \phi}, V^{u, \phi}(s), V_{(s)}^{u, \phi}\right) d s \\
& \quad-\int_{t}^{T} Z^{u, \phi}(s) d W(s), \\
& V^{u, \phi}(t)=V^{t, \phi}(t), \quad t \in[0, u),
\end{aligned}
$$

with

$$
\begin{aligned}
\widetilde{B}:= & \left(f_{s}+\lambda_{s}\right) V^{u, \phi}(s)-\pi_{s}-\theta_{s}\left(\bar{V}_{(s)}\right) \\
& -\alpha_{s}\left(f_{s}-c_{s}\right) \bar{V}_{(s)} .
\end{aligned}
$$

Thus, we are now dealing with a scheme, that is, the one represented by system (61), which is independent of the riskfree rate $r$, where the parameter $h$ mimes the role played by the risk-free rate $r$ in the classical Black-Scholes equation. For the latter setting, the following result holds.

Theorem 17. Let one consider the forward-backward delayed system (61), assuming both Assumptions 14 and the existence of positive constants $k_{\sigma}$ and $K_{\sigma}$ such that, for any $(t, \phi) \in[0, T] \times$ $\mathscr{C}$, the following holds:

$$
k_{\sigma}<\sigma(t, \phi)<K_{\sigma}
$$

Then, for every $(u, \phi) \in[0, T] \times \mathscr{C}$,

$$
V^{u, \phi}(t)=u\left(t, S^{u, \phi}\right), \quad \forall t \in[u, T],
$$

where $u(t, \phi)=V^{t, \phi}(t)$ is a viscosity solution of the following path-dependent PDE:

$$
\begin{gathered}
\partial_{t} u(t, \phi)+\frac{1}{2} \sigma^{2}(t, \phi) \partial_{x x}^{2} u(t, \phi)+r_{t} \partial_{x} u(t, \phi) \\
=\widetilde{B}\left(t, \phi, u(t, \phi), \partial_{x} u(t, \phi) \sigma(t, \phi),(u(\cdot, \phi))_{t}\right), \\
\phi \in \mathscr{C}, t \in[0, T), \\
u(T, \phi)=\Phi(\phi), \quad \phi \in \mathscr{C},
\end{gathered}
$$

with

$$
\begin{aligned}
\widetilde{B}(t, \phi, y, \widehat{y}):= & \left(f_{s}+\lambda_{s}\right) y-\pi_{s}-\theta_{s}(\widehat{y}) \\
& -\alpha_{s}\left(f_{s}-c_{s}\right) \hat{y} .
\end{aligned}
$$

Proof. The proof is analogous to the one provided for Theorem 16.

\section{Conclusion and Further Development}

Inspired by the increasing attention to financial models which take into account credit risk factors, we have generalized results provided in $[10,21]$ by exploiting techniques developed to treat backward stochastic differential equations (BSDEs).

Our BSDE approach generalizes the approach derived in $[10,21]$ allowing the coefficients $n$ the resulting pricing PDE to be possibly path-dependent. Moreover, as major novelty of our work, we are also able to treat a collateralization scheme that can depend on the past value of the contract.

We would like to underline the notion that the previous approach will be the base of our future works related to the fundamental topic of allowing for closeout rule with delay. Previous situation usually happens when one considers the time gap between the actual default of a party and the real 
closure of a contract. In such interval of time, it may happen that also the second party could default. Therefore, when one has to price a contract, such a time delay has to be taken into account; see, for example, [10] for a detailed treatment of the topic.

With respect to the aforementioned setting, we believe that both BSDEs techniques and the path-dependent calculus could turn out to be useful tools to treat the problem in concrete financial frameworks.

According to the recent literature, it is also important to note that the BSDEs techniques investigated in the present paper are also strongly connected to theoretical and, respectively, applied, questions (see, e.g., [43, 44]) that can lead to highly interesting developments, also from the statistical point of view.

\section{Competing Interests}

The authors declare that they have no competing interests regarding the publication of this paper.

\section{Acknowledgments}

The authors would like to thank the Gruppo Nazionale per l'Analisi Matematica, la Probabilità e le loro Applicazioni (GNAMPA), for the financial support that has funded the present research within the project called Set-Valued and Optimal Transportation Theory Methods to Model Financial Markets with Transaction Costs Both in Deterministic and Stochastic Frameworks.

\section{References}

[1] M. Henrard, Interest Rate Modelling in the Multi-Curve Framework: Foundations, Evolution and Implementation, Palgrave Macmillan, London, UK, 2014.

[2] N. Moreni and A. Pallavicini, "Parsimonious HJM modelling for multiple yield curve dynamics," Quantitative Finance, vol. 14, no. 2, pp. 199-210, 2014.

[3] A. Pallavicini and D. Brigo, "Interest-rate modelling in collateralized markets: multiple curves, credit-liquidity effects, CCPs," https://arxiv.org/abs/1304.1397.

[4] A. Pallavicini and M. Tarenghi, "Interest-rate modeling with multiple yield curves," SSRN Electronic Journal, 2010.

[5] Z. Grbac and W. J. Runggaldier, Interest Rate Modeling: PostCrisis Challenges and Approaches, SpringerBriefs in Quantitative Finance, Springer, 2015.

[6] Basel Committee on Banking Supervision-BI, "Principles for the Management of Credit Risk-Final Document," 2000.

[7] R. J. Elliott, M. Jeanblanc, and M. Yor, "On models of default risk," Mathematical Finance, vol. 10, no. 2, pp. 179-195, 2000.

[8] M. Jeanblanc and M. Rutkowski, "Default risk and hazard process," in Mathematical Finance-Bachelier Congress 2000, Springer, 2002.

[9] D. Brigo, M. Morini, and A. Pallavicini, Counterparty Credit Risk, Collateral and Funding: With Pricing Cases For All Asset Classes, John Wiley \& Sons, New York, NY, USA, 2013.

[10] D. Brigo and A. Pallavicini, "CCPs, central clearing, CSA, credit collateral and funding costs valuation FAQ: re-hypothecation,
CVA, closeout, netting, WWR, gap-risk, initial and variation margins, multiple discount curves, FVA?," https://arxiv.org/pdf/ 1312.0128.pdf.

[11] J. Liu, J. Xiao, L. Yan, and F. Wen, "Valuing catastrophe bonds involving credit risks," Mathematical Problems in Engineering, vol. 2014, Article ID 563086, 6 pages, 2014.

[12] A. Wang, L. Yong, W. Zeng, and Y. Wang, "The optimal analysis of default probability for a credit risk model," Abstract and Applied Analysis, vol. 2014, Article ID 878306, 9 pages, 2014.

[13] V. J. Leijdekker, M. R. Mandjes, and P. J. Spreij, "Sample-path large deviations in credit risk," Journal of Applied Mathematics, vol. 2011, Article ID 354171, 28 pages, 2011.

[14] L. Di Persio, M. Bonollo, and L. Prezioso, "Implicit trigger price determination for contingent convertible bond," International Journal of Pure and Apllied Mathematics, vol. 106, no. 3, 2016.

[15] F. Cordoni and L. Di Persio, "Invariant measure for the Vasicek interest rate model in the Heath-Jarrow-MortonMusiela frame-work," Infinite Dimensional Analysis Quantum Probability and Related Topics, vol. 18, no. 3, 2015.

[16] S. Crépey, "Bilateral counterparty risk under funding constraints-part I: pricing," Mathematical Finance, vol. 25, no. 1, pp. 1-22, 2015.

[17] S. Crépey, "Bilateral counterparty risk under funding constraints-part II: CVA," Mathematical Finance, vol. 25, no. 1, pp. 23-50, 2015.

[18] D. Brigo and A. Pallavicini, "CCP cleared or bilateral CSA trades with initial/variation margins under credit, funding and wrongway risks: a unified valuation approach," http://arxiv.org/abs/ 1401.3994.

[19] F. Cordoni and L. Di Persio, "Backward stochastic differential equations approach to hedging, option pricing, and insurance problems," International Journal of Stochastic Analysis, vol. 2014, Article ID 152389, 11 pages, 2014.

[20] N. El Karoui, S. Peng, and M. C. Quenez, "Backward stochastic differential equations in finance," Mathematical Finance, vol. 7, no. 1, pp. 1-71, 1997.

[21] D. Brigo, M. Francischello, and A. Pallavicini, "Invariance, existence and uniqueness of solutions of nonlinear valuation PDEs and FBSDEs inclusive of credit risk, collateral and funding costs," http://arxiv.org/abs/1506.00686.

[22] R. Cont and D.-A. Fournié, "Functional Itô calculus and stochastic integral representation of martingales," The Annals of Probability, vol. 41, no. 1, pp. 109-133, 2013.

[23] R. Cont and D.-A. Fournié, "Change of variable formulas for non-anticipative functionals on path space," Journal of Functional Analysis, vol. 259, no. 4, pp. 1043-1072, 2010.

[24] S.-E. A. Mohammed, "Stochastic differential systems with memory: theory, examples and applications," in Stochastic Analysis and Related Topics VI: Proceedings of the Sixth OsloSilivri Workshop Geilo 1996, L. Decreusefond, B. Øksendal, J. Gjerde, and A. S. Üstünel, Eds., vol. 42 of Progress in Probability, pp. 1-77, Birkhäuser, Boston, Mass, USA, 1998.

[25] B. Dupire, "Functional Itô calculus," Bloomberg Portfolio Research Paper 2009-04-FRONTIERS, 2009, http://papers.ssrn .com.

[26] D. R. Baños, F. Cordoni, G. Di Nunno, L. Di Persio, and E. E. Røse, "Stochastic systems with memory and jumps," https:// arxiv.org/abs/1603.00272.

[27] F. Cordoni, L. Di Persio, and I. Oliva, "A nonlinear Kolmogorov equation for stochastic functional delay differential equations with jumps," https://arxiv.org/abs/1602.03851. 
[28] A. Cosso and F. Russo, "A regularization approach to functional Itô calculus and strong-viscosity solutions to path-dependent PDEs," https://arxiv.org/abs/1401.5034vl.

[29] A. Cosso and F. Russo, "Functional Itô versus banach space stochastic calculus and strict solutions of semilinear pathdependent equations," http://arxiv.org/abs/1505.02926.

[30] F. Flandoli and G. Zanco, "An infinite-dimensional approach to path-dependent Kolmogorov's equations," http://arxiv.org/abs/ 1312.6165 .

[31] M. Fuhrman, F. Masiero, and G. Tessitore, "Stochastic equations with delay: optimal control via BSDEs and regular solutions of Hamilton-Jacobi-Bellman equations," SIAM Journal on Control and Optimization, vol. 48, no. 7, pp. 4624-4651, 2010.

[32] A. Swishchuk and L. Xu, "Pricing variance swaps for stochastic volatilities with delay and jumps," International Journal of Stochastic Analysis, vol. 2011, Article ID 435145, 27 pages, 2011.

[33] I. Ekren, C. Keller, N. Touzi, and J. Zhang, "On viscosity solutions of path dependent PDEs," The Annals of Probability, vol. 42, no. 1, pp. 204-236, 2014.

[34] I. Ekren, C. Keller, N. Touzi, and J. Zhang, "Viscosity solutions of fully nonlinear parabolic path dependent PDEs: part I," The Annals of Probability, In press.

[35] I. Ekren, N. Touzi, and J. Zhang, "Viscosity solutions of fully nonlinear parabolic path dependent PDEs: part II," https://arxiv .org/abs/1210.0007.

[36] S. Peng, "Nonlinear expectations, nonlinear evaluations and risk measures," in Stochastic Methods in Finance, vol. 1856 of Lecture Notes in Mathematics, pp. 165-253, Springer, Berlin, Germany, 2004.

[37] Ł. Delong and P. Imkeller, "Backward stochastic differential equations with time delayed generators-results and counterexamples," The Annals of Applied Probability, vol. 20, no. 4, pp. 1512-1536, 2010.

[38] F. Cordoni, L. Di Persio, L. Maticiuc, and A. Zălinescu, "A stochastic approach to path-dependent nonlinear Kolmogorov equations via BSDEs with time-delayed generators and applications to finance," http://arxiv.org/abs/1602.05793.

[39] V. Barbu, F. Cordoni, and L. Di Persio, "Optimal control of stochastic FitzHugh-Nagumo equation," International Journal of Control, vol. 89, no. 4, pp. 746-756, 2016.

[40] D. Brigo, A. Pallavicini, and V. Papatheodorou, "Arbitragefree valuation of bilateral counterparty risk for interest-rate products: impact of volatilities and correlations," International Journal of Theoretical and Applied Finance, vol. 14, no. 6, pp. 773802, 2011.

[41] T. R. Bielecki and M. Rutkowski, Credit Risk: Modeling, Valuation and Hedging, Springer Science \& Business Media, 2013.

[42] T. R. Bielecki, M. Jeanblanc-Picqué, and M. Rutkowski, Credit Risk Modeling, Osaka University Press, Osaka, Japan, 2009.

[43] C. Marinelli, L. Di Persio, and G. Ziglio, "Approximation and convergence of solutions to semilinear stochastic evolution equations with jumps," Journal of Functional Analysis, vol. 264, no. 12, pp. 2784-2816, 2013.

[44] L. Di Persio and M. Frigo, "Gibbs sampling approach to regime switching analysis of financial time series," Journal of Computational and Applied Mathematics, vol. 300, pp. 43-55, 2016. 


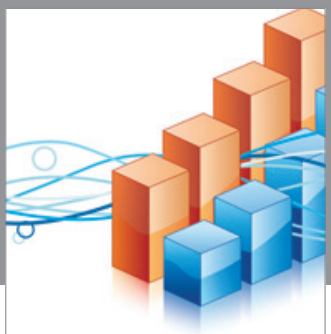

Advances in

Operations Research

vatem alat4

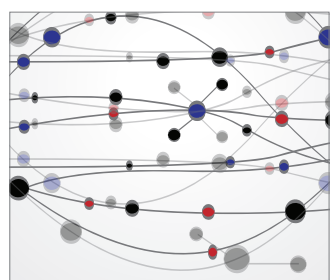

\section{The Scientific} World Journal
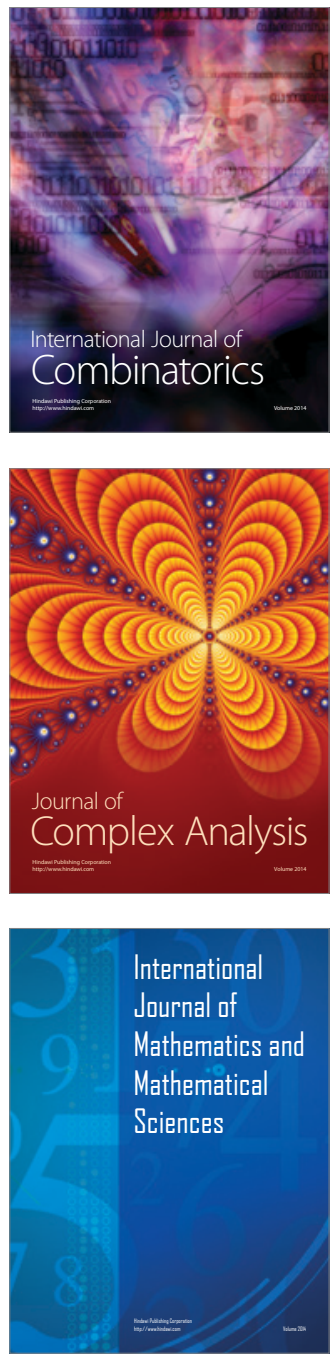
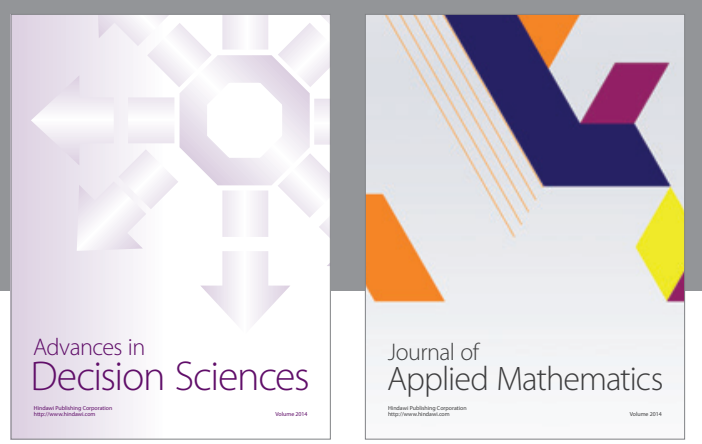

Algebra

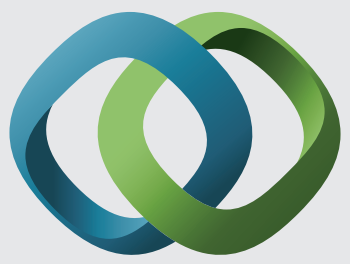

\section{Hindawi}

Submit your manuscripts at

http://www.hindawi.com
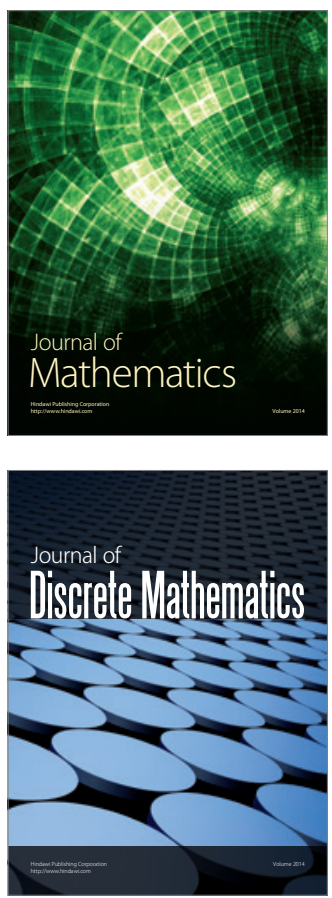

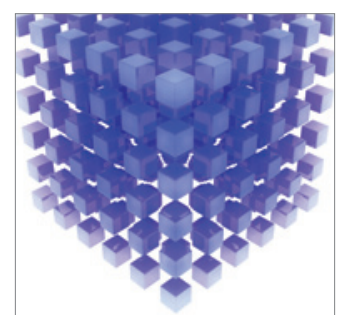

Mathematical Problems in Engineering
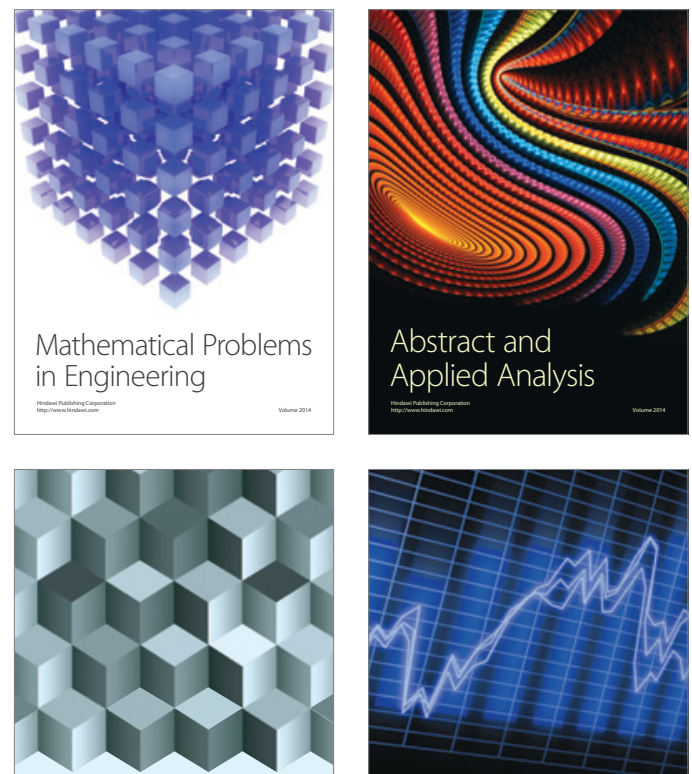

Journal of

Function Spaces

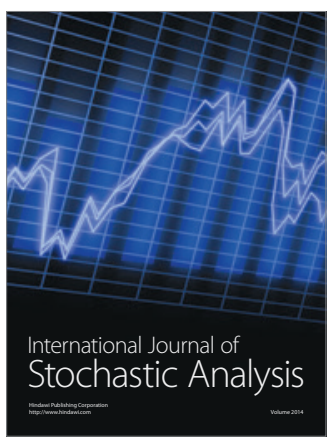

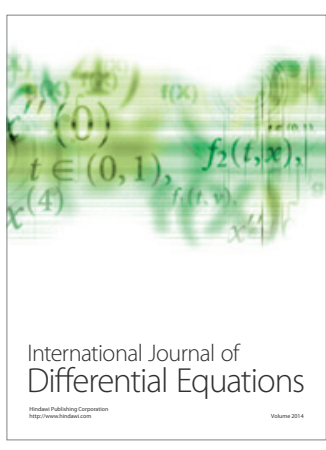
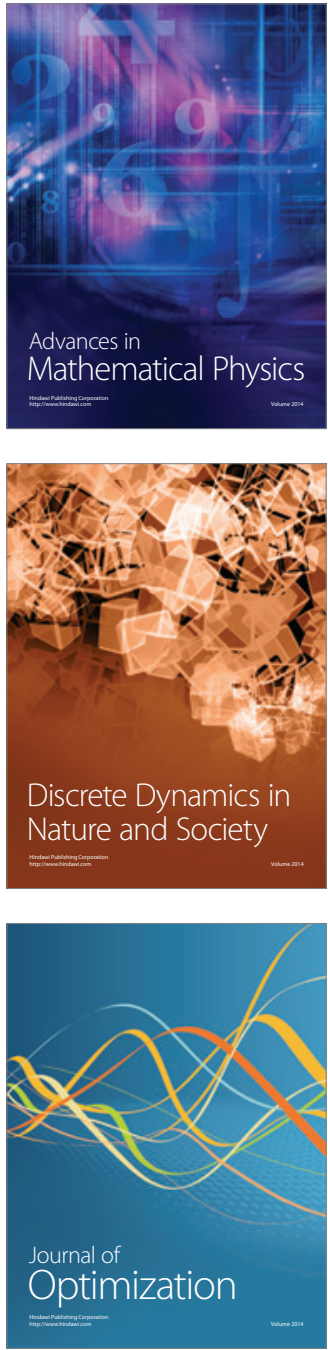